\title{
Nanoscale
}

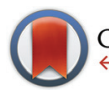

CrossMark \&lick for updates

Cite this: Nanoscale, 2016, 8, 8345

\section{Understanding the interaction between energetic ions and freestanding graphene towards practical 2D perforation $\dagger$}

\author{
Jakob Buchheim, ${ }^{a}$ Roman M. Wyss, ${ }^{a}$ Ivan Shorubalko*b and Hyung Gyu Park*a
}

We report experimentally and theoretically the behavior of freestanding graphene subjected to bombardment of energetic ions, investigating the capability of large-scale patterning of freestanding graphene with nanometer sized features by focused ion beam technology. A precise control over the $\mathrm{He}^{+}$and $\mathrm{Ga}^{+}$ irradiation offered by focused ion beam techniques enables investigating the interaction of the energetic particles and graphene suspended with no support and allows determining sputter yields of the 2D lattice. We found a strong dependency of the 2D sputter yield on the species and kinetic energy of the incident ion beams. Freestanding graphene shows material semi-transparency to $\mathrm{He}^{+}$at high energies (10-30 keV) allowing the passage of $>97 \% \mathrm{He}^{+}$particles without creating destructive lattice vacancy. Large $\mathrm{Ga}^{+}$ions (5-30 keV), in contrast, collide far more often with the graphene lattice to impart a significantly higher sputter yield of $\sim 50 \%$. Binary collision theory applied to monolayer and few-layer graphene can successfully elucidate this collision mechanism, in great agreement with experiments. Raman spectroscopy analysis corroborates the passage of a large fraction of $\mathrm{He}^{+}$ions across graphene without much damaging the lattice whereas several colliding ions create single vacancy defects. Physical understanding of the interaction between energetic particles and suspended graphene can practically lead to reproducible and efficient pattern generation of unprecedentedly small features on 2D materials by design, manifested by our perforation of sub-5 nm pore arrays. This capability of nanometer-scale precision patterning of freestanding $2 \mathrm{D}$ lattices shows the practical applicability of focused ion beam technology to 2D material processing for device fabrication and integration.

Received 7th January 2016, Accepted 17th March 2016 DOI: $10.1039 / \mathrm{c} 6 \mathrm{nr} 00154 \mathrm{~h}$ www.rsc.org/nanoscale synthesis methods such as chemical vapour deposition (CVD). ${ }^{9,10}$ Device integration of graphene to exploit its unique properties, on the other hand, will require selective patterning via etching or crystallographic modification through exposure to plasma ${ }^{11}$ or energetic ions. ${ }^{12}$ The evolution of the electrical properties and quality of supported or sandwiched graphene subjected to ion irradiation has been investigated, ${ }^{13-18}$ all confirming that graphene can be patterned by energetic ion irradiation. Nevertheless, it is the presence of a support structure that obscures a mechanistic understanding of the effects of ion bombardment on a $2 \mathrm{D}$ lattice of graphene because the bulk sputtering mechanism of the support ${ }^{19}$ perplexes the otherwise clearly observable 2D sputtering mechanism. For example, the effects of the secondary cascade interaction of bombarding particles with the support layer, such as ion implantation and substrate swelling, ${ }^{13,15,20}$ can influence experimental results significantly, eventually hampering the extraction of the 2D sputtering mechanism and the construction of fine features on a nanometer scale. On the other hand, freestanding graphene has been patterned using transmission electron microscopy where pores $^{21}$ and vacancies ${ }^{22}$ can be 
created with very high precision but are limited in scale due to immense irradiation dose required. Nanometer-sized feature formation in graphene has been enabled by block-copolymer self-assembly ${ }^{23}$ as well as by strain assisted metal intercalcination, ${ }^{24}$ both limited to feature sizes of $\sim 20 \mathrm{~nm}$. In the patternsize regime, other than these values, a focused ion beam (FIB) milling process of freestanding graphene can be a promising technology for practical applications at intermediate scales, considering that the FIB capability of nanoscale patterning under no such influence of substrates is adverse to fine feature-size control. Recently, the possibility of pattern generation in freestanding graphene by ion irradiation has been demonstrated by the formation of nanoscale pores, ${ }^{7,25}$ nanoribbons $^{26}$ or other geometries, ${ }^{27}$ though these studies lack a mechanistic understanding of the energetic-ion-graphene interaction. More insight into the interaction has been obtained by a few theoretical investigations using Monte Carlo simulations. ${ }^{17,18,28}$ Despite the growing understanding of the 2D sputtering mechanism including graphene amorphization upon $\mathrm{Ga}^{+}$irradiation, ${ }^{29}$ there are few reports of a combined experimental and theoretical investigation on the fate of freestanding graphene layers subjected to ion bombardment of various ion species and energies, hampering exploitation of the advanced manufacturing capability of the ion bombardment on freestanding graphene.

Here, we investigate experimentally and theoretically the effect of ion bombardment on freestanding graphene. The use of clean micrometer-wide suspended graphene allows us to determine quantitatively the probability and rates of carbon atom removal by avoiding substrate-caused ion sputtering artifacts. We differentiate interaction schemes of energetic ions with the graphene layer. Depending on their size and kinetic energy, ions can either penetrate the freestanding graphene without sputtering or collide with atoms of the $2 \mathrm{D}$ crystal to produce various vacancies. We rationalize these interactions by binary collision processes between incident ions and carbon atoms of the graphene lattice. In good agreement with experimental results, our model further elucidates the ion interaction mechanism with freestanding graphene. According to the results, interatomic lattice spacing of graphene can render it semi-transparent to incident energetic ions and tolerant to sputtering. The present work closes the gap between verification of theoretical predictions and practical formation of array patterns on freestanding graphene. An understanding of the nature of the energetic ion beam interaction with $2 \mathrm{D}$ materials of graphene enables pattern perforation on graphene by design, leading to an unprecedented dimension of reproducible graphene pore patterning via FIB milling such as sub-4 nm and sub-3 nm hole-arrays using $\mathrm{Ga}^{+}$and $\mathrm{He}^{+} \mathrm{FIB}$, respectively.

\section{Experimental}

\section{Freestanding graphene sample preparation}

Polycrystalline graphene was synthesized via CVD using conditions optimized for continued monolayer coverage reported elsewhere. ${ }^{30} \mathrm{~A} \mathrm{Cu}$ foil (Alfa Aesar 46986) is cleaned by Ar ion beam milling (10 $\mathrm{min}$ at $250 \mathrm{~mA}, 600 \mathrm{~V}$ acceleration) prior to a reduction annealing in a $\mathrm{H}_{2}$-rich atmosphere $\left(100 \mathrm{sccm} \mathrm{H}_{2}\right.$ in $1500 \mathrm{sccm} \mathrm{Ar}$ ) at $950{ }^{\circ} \mathrm{C}$ for $30 \mathrm{~min}$. Growth is initiated by the addition of $25 \mathrm{sccm}_{2} \mathrm{H}_{4}$ to the chamber for $2 \mathrm{~min}$ and subsequently $50 \mathrm{sccm}$ for $1 \mathrm{~min}$. The as-grown monolayer graphene was transferred onto a punctured $\operatorname{SiN}_{x}$ membrane supported by a Si chip (destination substrate) using a wet transfer method. ${ }^{7}$ PMMA is spun onto the graphene-coated $\mathrm{Cu}$ foil. $\mathrm{Cu}$ is etched away in $\left(\mathrm{NH}_{4}\right)_{2} \mathrm{~S}_{2} \mathrm{O}_{8}(0.5 \mathrm{M})$ leaving the graphene/PMMA film afloat the etchant. For multilayer graphene samples, this graphene/PMMA film is fished after rinsing in DI water with another graphene-grown $\mathrm{Cu}$ foil, and the additional $\mathrm{Cu}$ substrate is etched again. Repeating this step until the desired number of layers is reached, a clean multilayer graphene sample without interlayer contamination is obtained. After a final rinsing in DI water the floating graphene/PMMA film is fished with the porous SiNx destination substrate. Upon drying the graphene/PMMA film, the graphene is cleaned using well-known thermal decomposition methods ${ }^{31,32}$ where PMMA is pyrolyzed away at $400{ }^{\circ} \mathrm{C}$ in $900 \mathrm{sccm} \mathrm{H}_{2}$ and $100 \mathrm{sccm}$ Ar, yielding clean freestanding graphene.

\section{Graphene characterization}

The quality and cleanliness of the graphene samples were confirmed using scanning electron microscopy (Helios 450, FEI). Furthermore, the transferred graphene was analyzed before and after the patterning by 2D Raman mapping (micro Raman CRM200, WiTec) using a $532 \mathrm{~nm}$ incident laser beam at $0.4 \mathrm{~mW}$ with a pixel spacing of $100-250 \mathrm{~nm}$.

\section{Ion irradiation}

The freestanding graphene layers were irradiated with ions at different acceleration voltages via FIB (Helios 450, FEI). Toward the graphene sample $\mathrm{Ga}^{+}$ions are accelerated at 5-30 keV with probe currents ranging from $1.1 \mathrm{pA}$ to $40 \mathrm{pA}$ and a chamber pressure of $\sim 7 \times 10^{-5} \mathrm{~Pa}$. For the $\mathrm{He}^{+}$ion irradiation we used a $\mathrm{He}$ ion microscope (Zeiss Orion) equipped with a pattern generator (Raith Elphy MultiBeam) operated at 10-30 keV using a probe current of 5-17.5 pA at a chamber pressure of $\sim 7 \times 10^{-5} \mathrm{~Pa}$. Note that relatively high probe currents are desirable to reduce ion-beam-induced deposition in the exposed areas (ESI $\dagger$ ). On both tools the ion dose was controlled by the exposure dwell time of each pixel ranging from $100 \mathrm{~ns}$ to $8 \mathrm{~ms}$.

\section{Experimental sputter yield determination}

The sputter yield $(\gamma)$, defined as the average removal rate of carbon atoms from the graphene lattice, was determined by the following experiment. Circular pattern arrays of ions are accelerated toward freestanding graphene with increasing areal doses $(\sigma)$. On each circular pattern the energetic ions are evenly distributed with $200 \mathrm{~nm}$ in diameter (nominal area $A_{\mathrm{n}}=$ $0.0314 \mu \mathrm{m}^{2}$ ). After the patterning we acquire SEM micrographs and measure the resulting pattern area $A_{\mathrm{p}}$ using an image 
analysis program (ImageJ). The lower bound number of removed carbon atoms $N_{\mathrm{C}}=\sigma_{\mathrm{C}} A_{\mathrm{p}} N$ can be easily calculated by using the areal density of carbon in graphene $\sigma_{\mathrm{C}}\left(\mathrm{m}^{-2}\right)$ and the number of graphene layers, $N$. The sputter yield is defined as $\gamma_{\text {ion }}=N_{\mathrm{C}} / N_{\text {ion }}$, where $N_{\text {ion }}=\sigma_{\text {ion }} A_{\mathrm{n}}$ is the total number of ions irradiating the graphene layer.

\section{Graphene pore characterization}

The sizes of pore arrays in double layer graphene were determined using high-resolution SEM images obtained on a FEI Helios 450 at $5 \mathrm{keV}$ and $13 \mathrm{pA}$ probe current collecting secondary electrons. The micrograph was analyzed (ImageJ) by identifying the circular pore area by the dark regions (no secondary electrons generated), from which the diameter or the pore dimension was calculated. Electron micrographs of the smallest pores created in monolayer graphene were obtained using a Hitachi SU8230 SEM at $30 \mathrm{keV}$ and $55 \mathrm{pA}$ probe current and equipped with an SEM detector for a bright-field transmission electron mode (aperture size: $1 \mathrm{~mm}$ ). The pore area appears as a bright area where electrons pass the sample without being scattered. The micrographs were analyzed using the Gatan Digital Micrograph image analysis software.

\section{Results and discussion}

\section{Measurement of graphene sputter yield}

A high degree of control of three synthesis steps of graphene device fabrication (CVD graphene growth, transfer, and a subsequent annealing process) yielded ultraclean, freestanding graphene samples (Fig. 1a) showing very few graphene wrinkles and sparse contamination sites. These samples transferred to the FIB chamber were irradiated with energetic ions. When exposed to $\mathrm{He}^{+}$irradiation, graphene was resistant to a high dose of ions bombarding the monolayer. We could take images of freestanding monolayer graphene repeatedly at a standard imaging dose $\left(\sigma_{\mathrm{He}^{+}} \approx 10^{18} \mathrm{~m}^{-2}\right)$ of the helium ion microscope (HIM) without significant damage to the samples, in agreement with previous findings. ${ }^{33,34}$ The power of HIM and the cleanliness of the graphene samples allow us to clearly distinguish the layer numbers of freestanding multilayer graphene (Fig. 1b).

However, in the same experiment on a FIB system using $\mathrm{Ga}^{+}$ions, we found that the freestanding graphene quickly deteriorated and was etched away. These findings indicate that the interaction between $\mathrm{Ga}^{+}$and carbon in the graphene lattice is more destructive than that between $\mathrm{He}^{+}$and graphene; namely, $\mathrm{a} \mathrm{Ga}^{+}$ion has a higher probability to chop off carbon atoms from the $2 \mathrm{D}$ lattice than a $\mathrm{He}^{+}$ion. To precisely quantify the difference in carbon atom removal, the sputter yield is determined by patterning $200 \mathrm{~nm}$ circular features into monolayer graphene. The average number of vacancies produced per ion bombardment, or a sputter yield $\gamma$, shows a significantly higher value for $\mathrm{Ga}^{+}$(Fig. 2a). For instance, the sputter yield of freestanding graphene upon $30 \mathrm{keV} \mathrm{Ga}{ }^{+}$bombardment is about $47 \%\left(\gamma_{\mathrm{Ga}^{+}} \approx 47 \%\right)$, which confirms the qualitative find-
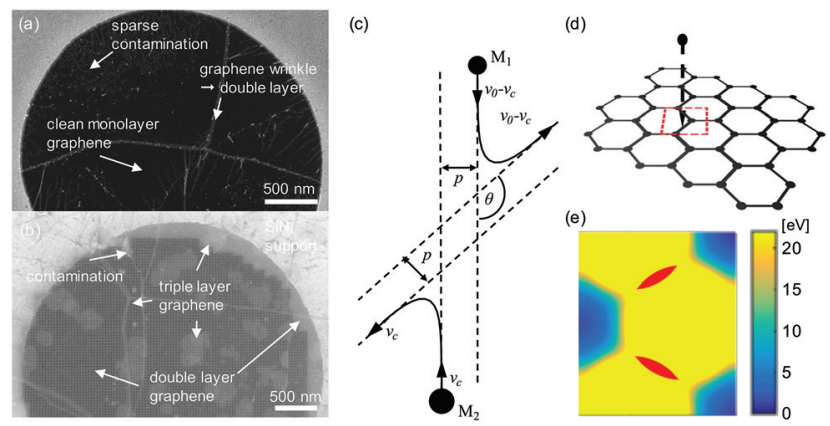

Fig. 1 (a) SEM micrograph of freestanding clean monolayer CVD graphene after transfer to porous $\mathrm{SiN}_{x}$ substrate. Brighter lines show graphene wrinkles (double layer) and small bright dots are sparse contaminates of graphene. (b) Helium ion micrograph of a $\mathrm{He}^{+}$patterned freestanding double layer graphene membrane. Local number variations of graphene layers can be clearly distinguished by pronounced brightness changes. (c) Binary collision model illustrated in a center-of-mass reference frame moving at a speed of $v_{c}$, where collision parameters $p$ and $\theta$ are the shortest projected distance and the scattering angle between two colliding particles, respectively (following ref. 19). (d) Schematic of the ion bombardment process of freestanding monolayer graphene. A red dashed box illustrates a unit cell of graphene. (e) A contour of superimposed energy from $15 \mathrm{keV} \mathrm{Ga}^{+}$to carbon atoms in a graphene unit cell, calculated from the binary collision model. The red shaded area depicts the area of double vacancy production.

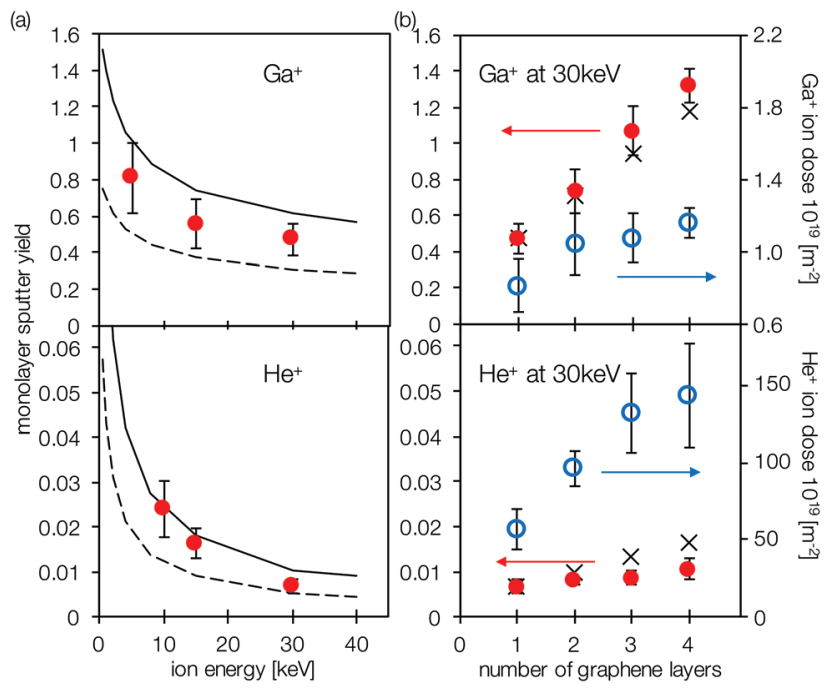

Fig. 2 (a) Energy dependent monolayer graphene sputter yields. Lower and upper bounds of theoretical sputter yield for $\mathrm{Ga}^{+}$and $\mathrm{He}^{+}$using a binary collision model (dashed black line and solid black line, respectively). Red dots are measured sputter yield for $\mathrm{Ga}^{+}$and $\mathrm{He}^{+}$ions, with error bars indicating the standard deviation. Each data point is calculated as the mean of more than 20 independent measurements. (b) Layer number dependent sputter yield and ion dose necessary to pattern graphene using $30 \mathrm{keV} \mathrm{Ga}^{+}$and $\mathrm{He}^{+}$ions. Red dots show sputter yield for 1-4 freestanding layers of graphene, and error bars indicate the standard deviation. Black crosses indicate the expected sputter yield assuming the layer number independent probability of carbon atom removal upon collision. The blue circle shows the experimentally measured ion dose for pattern formation. 
ings of previous reports on $\mathrm{Ga}^{+}$-based graphene sputtering. ${ }^{27,35}$ This corresponds to a total ion dose necessary to create a pattern in monolayer graphene by $30 \mathrm{keV} \mathrm{Ga}{ }^{+}$ions of $\sim 8.1 \times$ $10^{19} \mathrm{~m}^{-2}$ (Fig. 2b), which is very much in line with a recent report ${ }^{29}$ of $9.5 \times 10^{19} \mathrm{~m}^{-2}$ for $\mathrm{Ga}^{+}$at $35 \mathrm{keV}$. Furthermore, in our experiments $\gamma$ of freestanding monolayer graphene showed clear dependency on the energy of the incident particles. For $\mathrm{Ga}^{+}$the reduction of the accelerating voltage from $30 \mathrm{kV}$ to $5 \mathrm{kV}$ increases the sputter yield from $\gamma_{\mathrm{Ga}^{+}} \approx 47 \%$ to $81 \%$ (Fig. 2a), in line with predictions of Monte Carlo molecular dynamics simulations. ${ }^{17,18}$ Interestingly, these $\gamma_{\mathrm{Ga}^{+}}$values of monolayer graphene are significantly smaller than those of its 3D counterpart: reportedly 120-270\% depending on carbon allotropes. $^{35,36}$ For $\mathrm{He}^{+}$FIB the sputtering yield is about twoorders-of-magnitude lower $\left(\gamma_{\mathrm{He}^{+}} \approx 0.7 \%\right)$ than that for $\mathrm{Ga}^{+} \mathrm{FIB}$. Therefore, pattern generation with $30 \mathrm{keV} \mathrm{He}{ }^{+}$ions requires a significantly higher dose of $\sim 5.7 \times 10^{21} \mathrm{~m}^{-2}$ (Fig. 2b). Reaffirming the mechanism of HIM imaging, ${ }^{33,34}$ our finding sheds a renewed light on the possibility that, not only to protons, ${ }^{37,38}$ graphene can be nearly transparent to energetic $\mathrm{He}^{+}$ions as shown theoretically. ${ }^{17}$ For example, at a kinetic energy of $30 \mathrm{keV}$ approximately $99 \%$ of $\mathrm{He}^{+}$ions can penetrate through the monolayer graphene with statistically sputtering few or no carbon atoms from the lattice, reminiscent of the photon and proton transmission. The material transparency of graphene is slightly reduced at lower $\mathrm{He}^{+}$acceleration voltages $\left(\gamma_{\mathrm{He}^{+}}, 10 \mathrm{keV}\right.$ $\approx 2.4 \%$, Fig. 2 a) yet only to $\sim 97.6 \%$. Both observations of the material transmission of $\mathrm{H}^{+}$(ref. 37 and 38) and $\mathrm{He}^{+}$through the defect-free graphene lattice offers a new insight into the ability of graphene to serve as the barrier material. In the case of increased particle energy or commensurable particle size, graphene crystals in their freestanding state (let alone crystallographic defects) indeed allow the permeation of small atoms through the lattice.

\section{Calculation of theoretical sputter yield}

Since graphene is a 2D crystal of regularly bonded carbon atoms, its sputtering process can be assumed as a series of independent collision events between a bombarding ion and a lattice-bonded carbon atom individually. This interaction is then modeled by the use of the classic binary collision theory ${ }^{19,39}$ on the basis of an interception of two particle trajectories where the energetic ion at velocity $v_{0}$ collides with a carbon atom at a velocity $v_{\mathrm{c}}$ (Fig. 1c). Depending on the minimum projected distance between two particle trajectories, $p$, the nuclei start repelling each other to avoid overlap of the coulombic potential of the nuclei. The scatter angle, $\theta$, upon the interception (or collision) can be calculated using eqn (1):

$$
\theta(p)=\pi-2 \int_{r_{\min }}^{\infty} r^{-2}\left(1-V(r) / E_{\mathrm{C}}-p^{2} / r^{2}\right)^{-1 / 2} \mathrm{~d} r
$$

where $E_{\mathrm{C}}=E_{0} M_{2} /\left(M_{1}+M_{2}\right)$ denotes the collision energy with $E_{0}$ being the acceleration energy of the ion, $r$ the ion-to-atom center-of-mass distance, and $V(r)$ the Ziegler-BiersackLittmark interatomic potential between the ion and the atom
(Fig. 1c). A good approximation for the interatomic potential $V(r)=\frac{Z_{1} Z_{2} \mathrm{e}^{2}}{r} \Phi\left(\frac{r}{a}\right)$ is given by a repulsive coulombic potential created by the charges carried by the two nuclei corrected by a universal bfit function $\Phi(r / a)=\sum_{i=1}^{4} C_{i} \mathrm{e}^{k_{i} r / a}$ accounting for the electron cloud screening effects. The fit function depends on the distance of the scattering particles, $r$, the screening length, $a=0.8854 a_{0}\left(Z_{1}{ }^{0.23}+Z_{2}{ }^{0.23}\right)^{-1}$, calculated by the use of the Bohr radius, $a_{0}$, the two atomic charges $Z_{1}$ and $Z_{2}$ and has 8 parameters $C_{1}=0.1818, C_{2}=0.5099, C_{3}=0.2802, C_{4}=0.02811$ and $k_{1}=-3.2, k_{2}=-0.9423, k_{3}=-0.4029, k_{4}=-0.2016 .{ }^{19}$ It is valid for collisions with kinetic energies higher than a few $100 \mathrm{eV}$, where interatomic interactions are governed primarily by repulsive nuclei such that the Born-Oppenheimer approximation can be omitted. ${ }^{40}$ For each scatter angle, one can calculate the energy transferred, $T$, from the ion to the atom:

$$
T\left(E_{\mathrm{C}}, p\right)=4 E_{\mathrm{C}} \frac{M_{1}}{M_{1}+M_{2}} \sin \left(\frac{\theta(p)}{2}\right)^{2} .
$$

A carbon atom is removed from the graphene lattice if the transferred energy, $T\left(E_{\mathrm{C}}, p\right)$, exceeds the lattice displacement energy, $E_{\mathrm{L}}$. Previously reported $E_{\mathrm{L}}$ values for graphene range from experimentally determined $22 \mathrm{eV}$ (ref. 41) to density-functional-theory predicted $23 \mathrm{eV}^{42,43}$ Assuming $E_{\mathrm{L}}$ remains constant by neglecting vibrations of and locally altered binding energy of a defective graphene lattice in the course of sputter etching, we use this energy cut-off to calculate a theoretical sputter yield of graphene. Considering a graphene unit cell (Fig. 1d) with the superimposed transferred energy landscape around each carbon atom, we calculated the area fraction corresponding to $T\left(E_{\mathrm{C}}, p\right) \geq E_{\mathrm{L}}$ (yellow area) in which incident ions transfer energy higher than $E_{\mathrm{L}}$ to one carbon atom (Fig. 1e) for creating single vacancies, $\gamma_{\mathrm{s}}$. Moreover, an impact of an ion could produce a double vacancy, if the ion hits the unit cell in the area fraction, $\gamma_{\mathrm{d}}$, where the transferred energy to dual carbon atoms is higher than $E_{\mathrm{L}}$ (red area, Fig. 1e). The upper bound of the theoretical sputter yield, $\gamma_{\mathrm{U}}$, of a defect-free, relaxed graphene lattice can be calculated by $\gamma_{\mathrm{U}}=\gamma_{\mathrm{s}}+2 \gamma_{\mathrm{d}}$. Therefore, $\gamma_{\mathrm{U}}$ corresponds to the expectation value out of the discrete probability distribution of the following three events: ion passing without sputtering; producing a single vacancy defect; and producing a double vacancy defect.

\section{Comparison of experimental to theoretical sputter yield}

The calculated upper bound corresponds to the chance of carbon atom removal from a pristine monolayer graphene target and is necessarily higher than the experimental sputter yield which is an average removal rate in the course of graphene sputtering (Fig. 2a). Continued exposure to ions removes carbon atoms from the lattice, leading to lowered probability of bombarding carbon atoms by subsequent ion incidence, whose effect is manifested by a decrease in the sputter yield. Therefore, it is reasonable to define a lower bound of the theoretical sputter yield, $\gamma_{\mathrm{L}}$, by assuming an 
average probability of hitting a carbon atom accounting for already created vacancies through which ions may just pass the graphene lattice with no collision. For convenience, we ignore here the relaxation of the lattice once a carbon atom is chopped off, which could lead to a different lattice displacement energy for the subsequent sputtering events. Based on these assumptions we obtain $\gamma_{\mathrm{L}}=\gamma_{\mathrm{U}}\left(n_{\mathrm{C}}+1\right) / 2 n_{\mathrm{C}}$ (see the ESI $\dagger$ ), where $n_{\mathrm{C}}$ denotes the total number of ions directed toward graphene. For large values of $n_{\mathrm{C}}, \gamma_{\mathrm{L}}=\gamma_{\mathrm{U}} / 2$.

These two bounds set for the sputter yield can bracket our measured values greatly (Fig. 2a), suggesting that the measured sputter yield indeed corresponds to the sputtering events of clean monolayer graphene. The deviation of the measured yield from the upper bound is attributed to the gradual carbon atom removal from the target over the course of the ion exposure. In contrast the theoretical calculation of the lower bound does not include secondary carbon atom removal events such as potential removal of larger amorphous carbon atom agglomerates in the final phase of graphene etching. Furthermore, the excellent agreement within the employed energy range substantiates the validity of this collision theory in predicting sputter yields of graphene at various FIB conditions as well as in drawing a mechanistic explanation of the $\gamma$ values we obtained. Still, it would be worth considering an elaborate theoretical model to better compare with and explain the experimental observations.

Since the $\mathrm{Ga}^{+}$ions carry more charges in the nucleus than $\mathrm{He}^{+}$does, $V(r)$ with respect to a carbon atom can be stronger and extends wider in space. From eqn (1) and (2), this strongly repelling interatomic potential leads to large scattering angles close to the backscatter conditions, $\theta=\pi$, likely transferring a substantial amount of energy to an atom in the target lattice to chop it off. Besides capturing the different aspects of $\mathrm{He}^{+}$and $\mathrm{Ga}^{+}$sputtering, our model provides an accurate description about decreasing sputter yield of monolayer graphene with increasing kinetic energy of incident energetic ions (Fig. 2a), in good agreement with previous predictions. ${ }^{17,18}$ At lower kinetic energies the approaching ions get slower, interaction time prolongs, and the resultant scattering-angle distribution would become wider. Specifically, the interaction cross section extends wider in space, and since the average kinetic energy of the ions is still at least two-order-of-magnitude higher than $E_{\mathrm{L}}$, the bombarded atom in the lattice could possibly be removed upon, the results indicate that the repulsive interaction of the defect-free monolayer graphene becomes very strong when the energy of the colliding particle is comparable to or lower than the lattice displacement energy, corroborating the barrier properties of monolayer graphene previously reported. ${ }^{6}$ The good model fit, indicates that indeed the interactions between the ion and the graphene can be modeled as binary collisions where single carbon atoms are removed from the lattice when hit by the incoming ion. Therefore, previous theoretical findings are confirmed which show that in the energy range studied the indeed create only single or double vacancy defects. ${ }^{18}$ Only significantly lower energies $<200 \mathrm{eV}$ or $>50 \mathrm{keV}$ would lead to other effects like carbon atom substitution/ion implantation ${ }^{28}$ and graphene amorphization events in the vicinity of the impact position, ${ }^{18}$ respectively. Furthermore, the nature of the experimental design and the simplified modeling blur the effects of precise impact position on the carbon atom removal rate but gives a statistical average over all possible positions therefore corresponds to the realistic situation during graphene etching by energetic ions.

For multilayer graphene samples we observe an increase of the sputter yield (Fig. 2b). As a result, the ion dose necessary to carve out a pattern into freestanding multilayer graphene does not linearly increase with the number of layers (Fig. 2b). For example, a 4-layer graphene sample requires only $1.2 \times$ $10^{19} \mathrm{~m}^{-2} \mathrm{Ga}^{+}$ions at $30 \mathrm{keV}$ which roughly amount twice the dose required for a monolayer graphene. For $\mathrm{He}^{+}$ions the effect is less pronounced where thrice the dose of monolayer patterning is required to create a pattern in a 4-layer graphene sample (Fig. 2b). In general, results for freestanding multilayer graphene etching are approaching the sputter yield reported for bulk carbon allotropes. ${ }^{35,36}$ In sputtering bulk materials the so-called primary event - the collision of the incident ion with the target atom - does not play a crucial role in target atom removal. Though, the secondary sputtering events - the collisional cascade inside the target material leading to a momentum inversion - contribute mainly to the bulk sputter yield. The events responsible for the target atom removal in the 3D materials are the following: first, cascade of vibration energy into the target material could excite neighboring atoms in the bulk lattice; and second, these neighboring atoms recoil causing a local inversion of the momentum and could escape the bulk if they were close to the surface and ended up gaining sufficient energy. These events could often take place when the bulk material thicknesses are $\sim 20 \mathrm{~nm}$ and $\sim 5 \mathrm{~nm}$ for $\mathrm{Ga}^{+}$and $\mathrm{He}^{+}$, respectively. However, in the present case even the multilayer graphene samples are comparably thin having quadruple layers at most. Therefore, if we assume that only the probability of ions hitting a carbon atom should increase with the number of layers, then the sputtering of multilayer graphene can be approximated by independent stochastic events of collision with each graphene layer (providing that the kinetic energy of a scattered ion remains intact upon collision). On the other hand, the removal of carbon atoms in one layer would lower the probability that newly incident ions interact with atoms of the same layer since the number of layers at this particular spot is reduced by one. Therefore, the average expected sputter yield $\gamma_{N}$ for an $N$-layer-thick sample equals to $\gamma_{N}=\gamma_{\text {ion }}(N+1) / 2$. Using this assumption, we calculated an increase in sputter yields with layer number for both $\mathrm{He}^{+}$and $\mathrm{Ga}^{+}$FIB processes (Fig. 2b). For freestanding multilayer graphene, the experimentally observed sputter yield for $\mathrm{Ga}^{+}$ions increases from $\sim 0.5$ for monolayer graphene to $\sim 1.3$ for quadruple layer samples matching nicely the theoretical prediction (Fig. 2b). The consistency between experiment and theoretical prediction shows that interestingly the overall escape of the carbon atom from the multilayer graphene samples is not inhibited. Carbon atom removal in multilayer graphene could follow the route of a cascading collision of equal collision 
partners. Once a carbon atom in the first layers is hit it recoils and collides with a carbon atom in the lower layers from where the cascade continues until a carbon atom in the last graphene layer is removed by forward sputtering or even more complicated effects like catalytic etching in the presence of an underlying graphene layer ${ }^{41}$ may cause this counterintuitive finding. For $\mathrm{He}^{+}$ion the observed increase in sputter yield is less than the model prediction (Fig. 2b), possibly attributed to a less efficient vibrational cascade since the average transferred energy to the target atom is significantly lower than in the case of $\mathrm{Ga}^{+}$ion sputtering (see ESI $\dagger$ ). However, the presented analysis and experiments do not allow the detailed assessment of the fate of multilayer graphene on the atomic level. Density functional theory calculations state that the presence of additional graphene layers could significantly complicate the mechanism of vacancy formation in few-layer graphene. ${ }^{44}$ For example, a Frenkel defect in between adjacent graphene layers is prone to catalyzing divacancies and an intimate bi-Frenkel defect ${ }^{41,45}$ that interconnects these layers. ${ }^{46}$ These predictions suggest that, in contrast to interaction between monolayer graphene and energetic ion, the case of multiple graphene layers demands atomic-resolution imaging techniques and careful theoretical evaluation.

\section{Analysis of pattern formation by Raman spectroscopy}

Vacancy generation mechanism in graphene under energetic ion bombardment can be further elucidated by Raman spectroscopic monitoring of the evolution of pristine freestanding graphene subjected to various ion irradiation doses. 2D Raman maps are used to acquire a representative Raman signal from the pristine and irradiated graphene (Fig. 3a). The Raman spectrum of pristine graphene verifies high quality monolayer with a sharp G peak of FWHM $23.5 \mathrm{~cm}^{-1}$ at $\sim 1587 \mathrm{~cm}^{-1}$ (Fig. 3b), indicative of abundance of $\mathrm{sp}^{2}$-hybridized carbon atoms rather than $\mathrm{sp}^{3}{ }^{47}$ A sharp second harmonic benzene breathing mode commonly named a $\mathrm{G}^{\prime}$ peak around $\sim 2679 \mathrm{~cm}^{-1}$ shows a single peak of twice the intensity of the $\mathrm{G}$ peak (Fig. 3b), indicative of defect-free monolayer graphene. ${ }^{48}$ Defects in the graphene lattice can affect the intensity of the D band (at $\sim 1340 \mathrm{~cm}^{-1}$ ) in the Raman spectrum. This first harmonic or the radial breathing peak of the benzene ring unit activated in the case of asymmetry in a $\mathrm{sp}^{2}$-hybridized lattice $^{47}$ can originate largely from the vicinity of grain boundaries or vacancy sites and serves as a convenient measure of defect densities of any kind. In particular, the intensity ratio of the $\mathrm{D}$ and $\mathrm{G}$ peaks, $I(\mathrm{D}) / I(\mathrm{G})$, has been shown to follow a characteristic dependency on the defect density. ${ }^{49}$ Using the experimentally determined $\gamma$ and the areal dose, $\sigma$, of applied ions, we could calculate the average defect distance, $L_{\mathrm{D}}=$ $\left(\gamma_{\text {ion }} \sigma_{\text {ion }}\right)^{-1 / 2}$. The obtained result for $I(\mathrm{D}) / I(\mathrm{G})$ with respect to $L_{\mathrm{D}}$ for the $\mathrm{He}^{+}$FIB nicely matches an empirical equation previously reported ${ }^{49}$ about low energy $\mathrm{Ar}^{+}$bombardment (Fig. 3c). Indeed, this agreement corroborates our finding that a very high portion ( $>99 \%$ at $30 \mathrm{keV}$ ) of $\mathrm{He}^{+}$is passing through graphene without generating any lattice vacancy. It is the rare
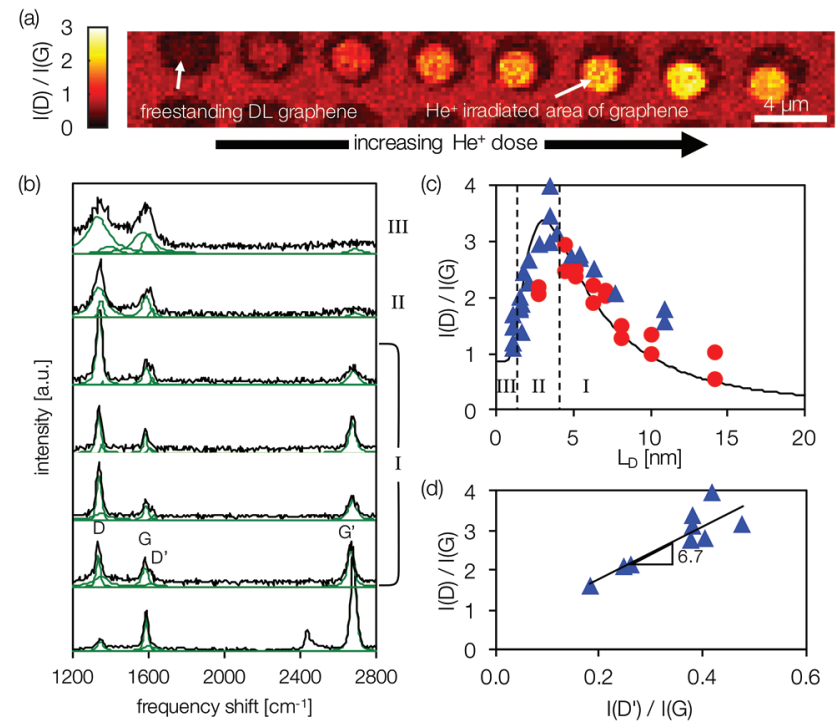

Fig. 3 Raman spectroscopic analysis of $\mathrm{He}^{+}$ion irradiated graphene. (a) 2D Raman map of $I(D) / /(G)$ on freestanding double layer graphene exposed to $\mathrm{He}^{+}$ion doses ranging from $0,2.5,3.8,5.0,6.3,9.4,12.5$ to $31.3 \times 10^{18} \mathrm{~m}^{-2}$. (b) Raman spectra of irradiated monolayer graphene with ion doses of $0,1.25,2.5,5,12.5,56,125 \times 10^{18} \mathrm{He}^{+} \mathrm{m}^{-2}$ (from bottom to top). (c) Measured $/(\mathrm{D}) / /(\mathrm{G})$ ratio versus average defect distance $L_{\mathrm{D}}$ for monolayer graphene (blue triangle) and double layer graphene (red circle) as compared with calculated empirical function (solid black line) from ref. 49. (d) $/(\mathrm{D}) / /(\mathrm{G})$ vs. $I\left(\mathrm{D}^{\prime}\right) / /(\mathrm{G})$ for monolayer graphene exposed to low $\mathrm{He}^{+}$ion doses $\left(1.25-62.5 \times 10^{18} \mathrm{~m}^{-2}\right)$, showing linear proportionality (solid black line).

collision events that produce a single vacancy defect on graphene.

The non-monotonic relation of $I(\mathrm{D}) / I(\mathrm{G})$ over the defect distance for freestanding graphene stands in contrast to the reported Tuinstra-Koenig relation reported for graphite. ${ }^{50}$ Unlike bulk graphite where ion irradiation renders the surface to $\mathrm{sp}^{3}$-bonded amorphous carbon yielding a monotonic increase of $I(\mathrm{D}) / I(\mathrm{G})$, ion irradiation of graphene exhibits three distinct regimes of etching. ${ }^{49}$ In the first regime (large $L_{\mathrm{D}}$ ), single vacancies are produced which lead to increased lattice disorder and a strong $\mathrm{D}$ peak. The total number of $\mathrm{sp}^{2}$ bonds remains nearly constant ( $G$ peak), yielding an increase of the $I(\mathrm{D}) / I(\mathrm{G})$ ratio (Fig. 3c, I). In the second regime $\left(L_{\mathrm{D}}<\sim 4 \mathrm{~nm}\right)$, the continuous removal of carbon atoms from the graphene layer destroys the hexagonal lattice, and the radial breathing (D peak) of benzene rings decreases in the abundance of intact benzene rings. Still, the layer comprises $\mathrm{sp}^{2}$-hybridized amorphous carbon chains accounting for the $G$ peak. In this regime $I(\mathrm{D}) / I(\mathrm{G})$ is getting smaller again (Fig. 3c, II). In the third regime $\left(L_{\mathrm{D}}<\sim 1 \mathrm{~nm}\right)$, the loose network of $\mathrm{sp}^{2}$-bonded carbon atoms is removed without significant changes in the bonding structure; therefore the $I(\mathrm{D}) / I(\mathrm{G})$ remains constant around unity with total peak intensities vanishing slowly until nearly all the atoms are removed (Fig. 3c, III).

The creation of individual vacancy defects in regime I during the $\mathrm{He}^{+}$FIB of graphene can be supported by spectral 
decomposition of Raman spectra. The evolution of $\mathrm{D}$ $\left(\sim 1340 \mathrm{~cm}^{-1}\right)$ versus $\mathrm{D}^{\prime}$ peaks $\left(\sim 1620 \mathrm{~cm}^{-1}\right)$ for low $\sigma_{\text {ion }}$ follows a linear proportionality of $I(\mathrm{D}) / I\left(\mathrm{D}^{\prime}\right) \approx 7$ (Fig. 3d) in good agreement with a previous report. ${ }^{51}$ This spectral behavior clearly differs from the other defect creation mechanisms such as $\mathrm{sp}^{3}$ bond creation or grain boundaries, which lead to $I(\mathrm{D}) / I\left(\mathrm{D}^{\prime}\right) \approx$ 13 and $I(\mathrm{D}) / I\left(\mathrm{D}^{\prime}\right) \approx 3.5$, respectively. ${ }^{51,52}$ Therefore, despite the low collision probability, irradiation of a low dose of energetic $\mathrm{He}^{+}$ions onto freestanding graphene could instigate a single ion graphene interaction facilitating single-vacancy-type defect generation in the lattice, preferentially. Furthermore, the resultant $I(\mathrm{D}) / I\left(\mathrm{D}^{\prime}\right)$ evolution shows clearly that the Raman spectrum does not arise from $\mathrm{sp}^{3}$ amorphous carbon deposits or contamination. Therefore, a precise control of $\sigma_{\mathrm{He}^{+}}$at a given $\mathrm{He}^{+}$energy shows promise to create array patterns of vacancy defects.

The evolution of the $I(\mathrm{D}) / I(\mathrm{G})$ ratio of double layer graphene follows the same trend as the monolayer graphene (solid red circles, Fig. 3c). When calculating the average distance $\left(L_{\mathrm{D}}\right)$ in which defects are created by considering the area of both graphene layers $L_{\mathrm{D}}=N^{1 / 2}\left(\gamma_{\mathrm{ion}} \sigma_{\text {ion }}\right)^{-1 / 2}$ (with $N=2$ ), the results for monolayer and double layer graphene coincide with each other, which corresponds to the case where the same number of ions directed toward graphene is distributed to twice the number of lattice atoms. At low defect densities $L_{\mathrm{D}}>5 \mathrm{~nm}$, the evolution of the $I(\mathrm{D}) / I(\mathrm{G})$ ratio versus $L_{\mathrm{D}}$ is almost the same as that on the monolayer, which indicates that the vacancy formation by energetic $\mathrm{He}^{+}$ions on double layer graphene is independent of the presence of a second layer. The individual Frenkel defects are presumably too far apart to be able to interact with each other, unlike what the DFT calculations predict. ${ }^{45}$ Therefore, we can confirm that each $\mathrm{He}^{+}$ion hitting the graphene either sputters one of the graphene layers or penetrates without creating a vacancy, and sputtering caused by the vibration energy cascade or by the graphene multilayer effect is nearly negligible.

Analysis of the Raman spectrum of ion-irradiated freestanding graphene elucidates the pattern formation on freestanding graphene via FIB. It follows the route of gradual vacancy formation at the initial step ensued by defect agglomeration that ends up amorphizing on the ion-beam irradiated area of the graphene lattice as recently proposed by a TEM study of irradiated graphene. ${ }^{29}$ In the last step of patterning the amorphous yet $\mathrm{sp}^{2}$-hybridized carbon layer is etched away.

\section{Critical dimension patterning of freestanding graphene}

Using these insights we could, for the first time, achieve the smallest pore-array patterns on graphene via FIB perforation. The 2D nature of graphene prefers forward sputtering such that each particle removal from the graphene lattice is caused by the particle collision with an incident energetic ion. On freestanding double layer graphene we cut holes with ultimate precision and repeatability at relatively high rates allowing an efficient large scale pattern formation. With $\sim 10^{4} \mathrm{Ga}^{+}$ions per pore at $30 \mathrm{keV}$ in a single-pixel exposure experiment, we could drill into double layer graphene an array of holes smaller than

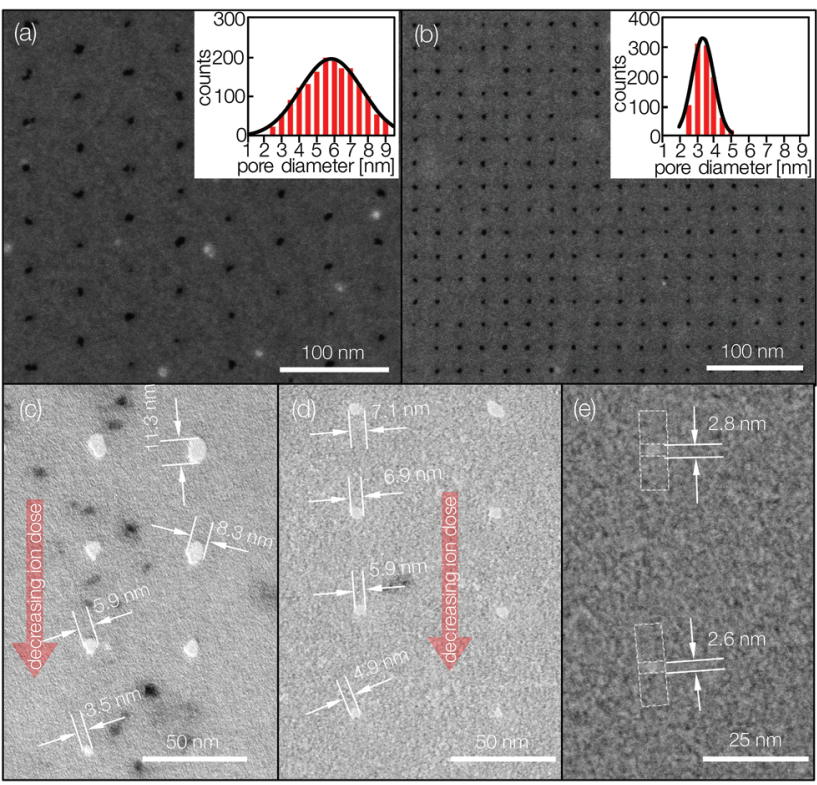

Fig. 4 Pore arrays on graphene perforated via FIB milling. Secondaryelectron-detection-mode SEM images of pore arrays having $50 \mathrm{~nm}$ wide (a) and $25 \mathrm{~nm}$ wide (b) pitches on freestanding double layer graphene perforated by $30 \mathrm{keV} \mathrm{Ga}^{+}$at $\sim 10^{4}$ per pore (a) and by $30 \mathrm{keV} \mathrm{He}^{+}$at $\sim 4.4$ $\times 10^{5}$ per pore (b), respectively. Dark contrast indicates pores. The resultant pore-size distributions are $5.9 \pm 3.6 \mathrm{~nm}$ (a) and very narrow, $3.3 \pm$ $1.2 \mathrm{~nm}$ (b). (c) Transmission-electron-mode (bright field) SEM image of pores on freestanding monolayer graphene perforated by $30 \mathrm{keV} \mathrm{Ga}^{+}$at $2500 \times 10^{4}$ per pore (bottom to top), exhibiting pore sizes from 3.5 to $11.3 \mathrm{~nm}$. (d, e) Transmission-electron-mode (bright field) SEM images of pores on the monolayer graphene perforated by $30 \mathrm{keV} \mathrm{He}{ }^{+}$at 3.9-6.2 $\times 10^{5}$ per pore (d, bottom to top) and $2.7 \times 10^{5} \mathrm{He}^{+}$per pore (e), showing pore sizes of 4.9-7.1 nm (d) and 2.6 and $2.8 \mathrm{~nm}$ (e), respectively.

$9 \mathrm{~nm}$ (with a mean diameter of $5.8 \mathrm{~nm}$ ) at the average spacing of $50 \mathrm{~nm}$ (Fig. 4a).

The critical dimension (CD) of the FIB-drilled pores is dependent on the ion irradiation dose. In order to obtain the relationship between $\mathrm{CD}$ and ion dose unambiguously, we characterized the FIB perforation of a simple monolayer graphene system. When the $30 \mathrm{keV} \mathrm{Ga}{ }^{+}$irradiation dose was reduced from $10^{4}$ to 7500 to 5000 to 2500 ions per pixel (or pore), the respective pore sizes measured 11.3, 8.3, 5.9 and $3.5 \mathrm{~nm}$ (Fig. 4c), revealing the dependency of the (monolayer) graphene $\mathrm{CD}$ on the ion dose. The smallest pore size obtained in this experiment is significantly smaller than the previously reported results of sub-10 nm pores on graphene. ${ }^{25}$ The tight control of the exposed ions does not only allow us to pattern at the resolution limit of the $\mathrm{Ga}^{+}$FIB system defined by the beam size $(\sim 4 \mathrm{~nm})$. The low dose guaranties an exceptionally short process time of a few microseconds per pattern, enabling even large-scale patterning of graphene for device integration.

Note that these top-down-drilled pore sizes are significantly smaller than sputtering of bulk material could produce. In the 3D sputtering process, an incident ion collides with multiple target atoms to initiate a collision cascade within the target 
material. Recoiling target atoms can induce a momentum inversion of certain atoms close to the surface, resulting in an escape from the bulk phase. ${ }^{19}$ These secondary events occur in the vicinity of the incident ion spot called an interaction diameter, the reported values of which are around $20-30 \mathrm{~nm} .^{53,54}$

For ion exposure of graphene in HIM we achieved even smaller feature sizes. Here again we could overcome the previously reported interaction diameter limit of $5 \mathrm{~nm}$ (ref. 53) and repeatedly pattern pore arrays into freestanding double layer graphene with a mean diameter of $3.4 \mathrm{~nm}$ at $25 \mathrm{~nm}$ wide spacing using $30 \mathrm{keV} \mathrm{He}^{+}$ions at $4.4 \times 10^{5}$ per pore (Fig. $4 \mathrm{~b}$ ). The effect of the total ion dose on CD (the pore size) can be observed by exposing freestanding monolayer graphene to 3.9-6.2 $\times 10^{5} \mathrm{He}^{+}$ions per pixel, revealing again the ion dose dependency of the (monolayer) graphene CD (Fig. 4d).

Despite the single-pixel exposure we see a pore size increase from 4.9 to $7.1 \mathrm{~nm}$ caused by the imperfect spot shape of the irradiating beam. By decreasing the number of $\mathrm{He}^{+}$hitting the monolayer graphene to $2.7 \times 10^{5}$ we were able to produce holes with $2.6 \mathrm{~nm}$ wide diameter (Fig. 4e), comparable to pore sizes drilled in graphene using TEM systems. ${ }^{21,55}$ Our results show a significant advancement in graphene patterning via FIB milling in terms of feature size and array dimension, enabled by detailed knowledge of the interaction mechanisms involved and the 2D nature of our target material. The use of freestanding graphene allowed us to create patterns while avoiding undesirable secondary effects during FIB milling (e.g., ion implanting and substrate swelling), unlike those frequently reported for the supported graphene samples. ${ }^{13,15,35,56}$

\section{Conclusions}

Experimental and theoretical investigations of the interaction between freestanding graphene layers and energetic ion irradiation confirm that pristine graphene could be transparent to materials at elevated kinetic energy, suggested by previous theoretical investigations. ${ }^{17}$ For instance, graphene is highly transparent to $30 \mathrm{keV}$-accelerated $\mathrm{He}^{+}$particles, only $\sim 1 \%$ of which collide with the graphene lattice and sputter carbon atoms as compared with $47 \%$ for $\mathrm{Ga}^{+}(30 \mathrm{keV})$. Both binary collision theory and experimental characterization point out the uniqueness of $2 \mathrm{D}$ material sputtering in that the major sputtering mechanism would be a simple binary collision between the incident ion particle and the carbon atom in the lattice, in clear contrast to vibration energy cascade and recoiling for $3 \mathrm{D}$ material sputtering and also in contrast to the DFT theory predictions on the multilayer effect. These findings represent a simple understanding of ballistic ion collisions with $2 \mathrm{D}$ materials and permit the prediction of an actual sputter yield of atomically thin targets, which has not been possible with other techniques. The sputtering probability (i.e., sputter yield) of the freestanding graphene layers depends strongly on the species and kinetic energies of the bombarding ion particles, as well as on the number of layers. These findings lead altogether to a rationale for the 2D material pattern- ing such that precise control of the local ion irradiation dose on freestanding 2D materials could bring the size limit of this technology to a new level. This pattern generation strategy was firstly attested by the creation of hole-array patterns with feature sizes down to $2.6 \mathrm{~nm}$ and $3.5 \mathrm{~nm}$ using $\mathrm{He}^{+} \mathrm{FIB}$ and $\mathrm{Ga}^{+}$FIB, respectively. These results highlight the great potential of efficient, sub-5 $\mathrm{nm}$ scale feature generation on $2 \mathrm{D}$ materials and thereby close the gap between theory verification and pore-array-patterning practice, opening up great possibilities in the nanomanufacture of devices that use flexible $2 \mathrm{D}$ materials patterned on a nanometer scale.

\section{Acknowledgements}

We appreciate the support from Binnig and Rohrer Nanotechnology Center of ETH Zurich and IBM Zurich. J.B. thanks the Swiss National Science Foundation for financial support (200021-137964). I.S. is grateful to the Swiss National Science Foundation for support in equipment procurement (REquip 206021-133823). This work was partially supported by LG Electronics Advanced Research Institute, for which R.M.W. is thankful. I.S. thanks Y. Zheng at EMPA (Swiss Federal Laboratories for Materials Science and Technology), Switzerland, for his initial contribution to the perforated graphene imaging.

\section{References}

1 K. S. Novoselov, V. I. Fal'ko, L. Colombo, P. R. Gellert, M. G. Schwab and K. Kim, Nature, 2012, 490, 192-200.

2 C. Lee, X. Wei, J. W. Kysar and J. Hone, Science, 2008, 321, 385-388.

3 K. S. Novoselov, A. K. Geim, S. V. Morozov, D. Jiang, Y. Zhang, S. V. Dubonos, I. V. Grigorieva and A. A. Firsov, Science, 2004, 306, 666-669.

4 A. A. Balandin, S. Ghosh, W. Bao, I. Calizo, D. Teweldebrhan, F. Miao and C. N. Lau, Nano Lett., 2008, 8, 902-907.

5 R. R. Nair, P. Blake, A. N. Grigorenko, K. S. Novoselov, T. J. Booth, T. Stauber, N. M. Peres and A. K. Geim, Science, 2008, 320, 1308.

6 J. S. Bunch, S. S. Verbridge, J. S. Alden, A. M. van der Zande, J. M. Parpia, H. G. Craighead and P. L. McEuen, Nano Lett., 2008, 8, 2458-2462.

7 K. Celebi, J. Buchheim, R. M. Wyss, A. Droudian, P. Gasser, I. Shorubalko, J. I. Kye, C. Lee and H. G. Park, Science, 2014, 344, 289-292.

8 L. Peng, X. Peng, B. Liu, C. Wu, Y. Xie and G. Yu, Nano Lett., 2013, 13, 2151-2157.

9 X. Li, W. Cai, J. An, S. Kim, J. Nah, D. Yang, R. Piner, A. Velamakanni, I. Jung, E. Tutuc, S. K. Banerjee, L. Colombo and R. S. Ruoff, Science, 2009, 324, 1312-1314.

10 S. Bae, H. Kim, Y. Lee, X. Xu, J. S. Park, Y. Zheng, J. Balakrishnan, T. Lei, H. R. Kim, Y. I. Song, Y. J. Kim, 
K. S. Kim, B. Ozyilmaz, J. H. Ahn, B. H. Hong and S. Iijima, Nat. Nanotechnol., 2010, 5, 574-578.

11 T. Gokus, R. R. Nair, A. Bonetti, M. Bohmler, A. Lombardo, K. S. Novoselov, A. K. Geim, A. C. Ferrari and A. Hartschuh, ACS Nano, 2009, 3, 3963-3968.

12 J. H. Chen, W. G. Cullen, C. Jang, M. S. Fuhrer and E. D. Williams, Phys. Rev. Lett., 2009, 102, 236805.

13 M. C. Lemme, D. C. Bell, J. R. Williams, L. A. Stern, B. W. Baugher, P. Jarillo-Herrero and C. M. Marcus, ACS Nano, 2009, 3, 2674-2676.

14 B. S. Archanjo, A. P. Barboza, B. R. Neves, L. M. Malard, E. H. Ferreira, J. C. Brant, E. S. Alves, F. Plentz, V. Carozo, B. Fragneaud, I. O. Maciel, C. M. Almeida, A. Jorio and C. A. Achete, Nanotechnology, 2012, 23, 255305.

15 A. N. Abbas, G. Liu, B. Liu, L. Zhang, H. Liu, D. Ohlberg, W. Wu and C. Zhou, ACS Nano, 2014, 8, 1538-1546.

16 G. Nanda, S. Goswami, K. Watanabe, T. Taniguchi and P. F. Alkemade, Nano Lett., 2015, 15, 4006-4012.

17 O. Lehtinen, J. Kotakoski, A. V. Krasheninnikov, A. Tolvanen, K. Nordlund and J. Keinonen, Phys. Rev. B: Condens. Matter, 2010, 81, 153401.

18 O. Lehtinen, J. Kotakoski, A. V. Krasheninnikov and J. Keinonen, Nanotechnology, 2011, 22, 175306.

19 J. F. Ziegler, J. P. Biersack and U. Littmark, The Stopping and Range of Ions in Solids, Pergamon Press, 1985.

20 D. C. Bell, M. C. Lemme, L. A. Stern, J. R. Williams and C. M. Marcus, Nanotechnology, 2009, 20, 455301.

21 M. D. Fischbein and M. Drndic, Appl. Phys. Lett., 2008, 93, 113107.

22 A. W. Robertson, C. S. Allen, Y. A. Wu, K. He, J. Olivier, J. Neethling, A. I. Kirkland and J. H. Warner, Nat. Commun., 2012, 3, 11440.

23 J. Bai, J. Bai, X. Zhong, X. Zhong, S. Jiang, S. Jiang, Y. Huang, Y. Huang, X. Duan and X. Duan, Nat. Nanotechnol., 2010, 5, 190-194.

24 S.-S. Kim, M. J. Park, J.-H. Kim, G. Ahn, S. Ryu, B. H. Hong and B.-H. Sohn, Chem. Mater., 2015, 27, 7003-7010.

25 A. Hemamouche, A. Morin, E. Bourhis, B. Toury, E. Tarnaud, J. Mathe, P. Guegan, A. Madouri, X. Lafosse, C. Ulysse, S. Gullet, G. Patriarche, L. Auvray, F. Montel, Q. Wilmart, B. Placais, J. Yates and J. Gierak, Microelectron. Eng., 2014, 121, 87-91.

26 D. Pickard and L. Scipioni, Zeiss application note, 2009.

27 D. Lucot, J. Gierak, A. Ouerghi, E. Bourhis, G. Faini and D. Mailly, Microelectron. Eng., 2009, 86, 882-884.

28 E. H. Åhlgren, J. Kotakoski and A. V. Krasheninnikov, Phys. Rev. B: Condens. Matter, 2011, 83, 115424.

29 J. Kotakoski, C. Brand, Y. Lilach, O. Cheshnovsky, C. Mangler, M. Arndt and J. C. Meyer, Nano Lett., 2015, 15, 5944-5949.

30 K. Celebi, M. T. Cole, J. W. Choi, F. Wyczisk, P. Legagneux, N. Rupesinghe, J. Robertson, K. B. Teo and H. G. Park, Nano Lett., 2013, 13, 967-974.

31 Z. Cheng, Q. Zhou, C. Wang, Q. Li, C. Wang and Y. Fang, Nano Lett., 2011, 11, 767-771.
32 S. Průša, P. Procházka, P. Bábor, T. Šikola, R. ter Veen, M. Fartmann, T. Grehl, P. Brüner, D. Roth, P. Bauer and H. H. Brongersma, Langmuir, 2015, 31, 9628-9635.

33 D. Fox, Y. B. Zhou, A. O'Neill, S. Kumar, J. J. Wang, J. N. Coleman, G. S. Duesberg, J. F. Donegan and H. Z. Zhang, Nanotechnology, 2013, 24, 335702.

34 Y. Zhou, R. O’Connell, P. Maguire and H. Zhang, Sci. Rep., 2014, 4, 7032.

35 J. J. Lopez, F. Greer and J. R. Greer, J. Appl. Phys., 2010, 107, 104326.

36 A. Stanishevsky, Thin Solid Films, 2001, 398, 560-565.

37 S. Hu, M. Lozada-Hidalgo, F. C. Wang, A. Mishchenko, F. Schedin, R. R. Nair, E. W. Hill, D. W. Boukhvalov, M. I. Katsnelson, R. A. W. Dryfe, I. V. Grigorieva, H. A. Wu and A. K. Geim, Nature, 2014, 516, 227-230.

38 S. Mathew, T. K. Chan, D. Zhan, K. Gopinadhan, A. R. Barman, M. B. H. Breese, S. Dhar, Z. X. Shen, T. Venkatesan and J. T. L. Thong, Carbon, 2011, 49, 17201726.

39 G. C. Maitland, M. Rigby, E. B. Smith and W. A. Wakeham, Intermolecular Forces: Their Origin and Determination, Clarendon Press, 1981.

40 J. Burgdörfer, Atomic Collisions with Surfaces, World Scientific Publishing, 1993.

41 J. Zubeltzu, A. Chuvilin, F. Corsetti, A. Zurutuza and E. Artacho, Phys. Rev. B: Condens. Matter, 2013, 88, 245407.

42 A. V. Krasheninnikov, F. Banhart, J. X. Li, A. S. Foster and R. M. Nieminen, Phys. Rev. B: Condens. Matter, 2005, 72, 125428.

43 A. Zobelli, A. Gloter, C. P. Ewels, G. Seifert and C. Colliex, Phys. Rev. B: Condens. Matter, 2007, 75, 245402.

44 F. Banhart, J. Kotakoski and A. V. Krasheninnikov, ACS Nano, 2011, 5, 26-41.

45 R. H. Telling, C. P. Ewels, A. A. El-Barbary and M. I. Heggie, Nat. Mater., 2003, 2, 333-337.

46 C. P. Ewels, R. H. Telling, A. A. El-Barbary, M. I. Heggie and P. R. Briddon, Phys. Rev. Lett., 2003, 91, 025505.

47 A. C. Ferrari and D. M. Basko, Nat. Nanotechnol., 2013, 8, 235-246.

48 A. C. Ferrari, J. C. Meyer, V. Scardaci, C. Casiraghi, M. Lazzeri, F. Mauri, S. Piscanec, D. Jiang, K. S. Novoselov, S. Roth and A. K. Geim, Phys. Rev. Lett., 2006, 97, 187401.

49 M. M. Lucchese, F. Stavale, E. H. M. Ferreira, C. Vilani, M. V. O. Moutinho, R. B. Capaz, C. A. Achete and A. Jorio, Carbon, 2010, 48, 1592-1597.

50 F. Tuinstra and J. L. Koenig, J. Chem. Phys., 1970, 53, 11261130.

51 A. Eckmann, A. Felten, A. Mishchenko, L. Britnell, R. Krupke, K. S. Novoselov and C. Casiraghi, Nano Lett., 2012, 12, 3925-3930.

52 A. Eckmann, A. Felten, I. Verzhbitskiy, R. Davey and C. Casiraghi, Phys. Rev. B: Condens. Matter, 2013, 88, 035426.

53 M. Ananth, L. Stern, D. Ferranti, C. Huynh, J. Notte, L. Scipioni, C. Sanford and B. Thompson, Scanning 
Microscopies 2011: Advanced Microscopy Technologies for Defense, Homeland Security, Forensic, Life, Environmental, and Industrial Sciences, 2011, vol. 8036, p. 80360M.

54 C. J. Lo, T. Aref and A. Bezryadin, Nanotechnology, 2006, 17, 56 S. J. Hang, Z. Moktadir and H. Mizuta, Carbon, 2014, 72, 3264-3267.
55 G. F. Schneider, Q. Xu, S. Hage, S. Luik, J. N. H. Spoor, S. Malladi, H. Zandbergen and C. Dekker, Nat. Commun., 2013, 4, 2619. 233-241. 